J. Nonlinear Var. Anal. 3 (2019), No. 2, pp. 159-170

Available online at http://jnva.biemdas.com

https://doi.org/10.23952/jnva.3.2019.2.05

\title{
WEAK CONVERGENCE THEOREMS FOR INFINITE FAMILIES OF EXTENDED GENERALIZED HYBRID MAPPINGS IN BANACH SPACES
}

\author{
MAYUMI HOJO
}

Shibaura Institute of Technology, 307 Fukasaku, Minuna-ku, Saitama 337-8570, Japan

Abstract. Let $E$ be a real Banach space and let $C$ be a nonempty subset of $E$. A mapping $T: C \rightarrow E$ is said to be extended generalized hybrid if there are $\alpha, \beta, \gamma, \delta \in \mathbb{R}$ such that $\alpha+\beta+\gamma+\delta \geq 0, \alpha+\beta>0$ and

$$
\alpha\|T x-T y\|^{2}+\beta\|x-T y\|^{2}+\gamma\|T x-y\|^{2}+\delta\|x-y\|^{2} \leq 0
$$

for all $x, y \in C$. In this paper, we prove a weak convergence theorem of Mann's type iteration for infinite families of extended generalized hybrid mappings in a Banach space satisfying the Opial's condition.

Keywords. Banach space; Extended generalized hybrid mapping; Fixed point; Opial's condition; Weak convergence theorem. 2010 Mathematics Subject Classification. 47H10, 47H05.

\section{INTRODUCTION}

Let $H$ be a real Hilbert space and let $C$ be a nonempty subset of $H$. A mapping $T: C \rightarrow H$ is said to be nonexpansive if $\|T x-T y\| \leq\|x-y\|$ for all $x, y \in C$. In 2010, Kocourek, Takahashi and Yao [11] defined a broad class of nonlinear mappings in a Hilbert space which covers nonexpansive mappings: Let $C$ be a nonempty subset of $H$. A mapping $T: C \rightarrow H$ is called generalized hybrid [11] if there exist $\alpha, \beta \in \mathbb{R}$ such that

$$
\alpha\|T x-T y\|^{2}+(1-\alpha)\|x-T y\|^{2} \leq \beta\|T x-y\|^{2}+(1-\beta)\|x-y\|^{2}
$$

for all $x, y \in C$. Such a mapping $T$ is called $(\alpha, \beta)$-generalized hybrid. We also know the following fact. For $\lambda \in \mathbb{R}$, a mapping $U: C \rightarrow H$ is called $\lambda$-hybrid [1] if

$$
\|U x-U y\|^{2} \leq\|x-y\|^{2}+2(1-\lambda)\langle x-U x, y-U y\rangle
$$

for all $x, y \in C$. Notice that the class of generalized hybrid mappings covers several well-known mappings in a Hilbert space. For example, a $(1,0)$-generalized hybrid mapping is nonexpansive. It is nonspreading $[12,13]$ for $\alpha=2$ and $\beta=1$, i.e.,

$$
2\|T x-T y\|^{2} \leq\|T x-y\|^{2}+\|T y-x\|^{2}, \quad \forall x, y \in C .
$$

It is also hybrid [18] for $\alpha=\frac{3}{2}$ and $\beta=\frac{1}{2}$, i.e.,

$$
3\|T x-T y\|^{2} \leq\|x-y\|^{2}+\|T x-y\|^{2}+\|T y-x\|^{2}, \quad \forall x, y \in C .
$$

E-mail address: mayumi-h@ shibaura-it.ac.jp.

Received February 19, 2019; Accepted April 28, 2019.

(C)2019 Journal of Nonlinear and Variational Analysis 
In general, nonspreading and hybrid mappings are not continuous; see [9]. We also know that $\lambda$-hybrid mappings in a Hilbert space are contained in the class of generalized hybrid mappings; see [8]. Hojo and Takahashi [6] extended the concept of generalized hybrid mappings in a Hilbert space to that in a Banach space as follows: Let $E$ be a Banach space and let $C$ be a nonempty subset of $E$. A mapping $T: C \rightarrow E$ is called extended generalized hybrid [6] if there are $\alpha, \beta, \gamma, \delta \in \mathbb{R}$ such that $\alpha+\beta+\gamma+\delta \geq 0, \alpha+\beta>0$ and

$$
\alpha\|T x-T y\|^{2}+\beta\|x-T y\|^{2}+\gamma\|T x-y\|^{2}+\delta\|x-y\|^{2} \leq 0
$$

for all $x, y \in C$. We call such a mapping $(\alpha, \beta, \gamma, \delta)$-extended generalized hybrid. Hojo and Takahashi [7] proved the following weak convergence theorem for finding a common fixed point of two extended generalized hybrid mappings in a Banach space by using Mann's type iteration [14]; see also [19].

Theorem 1.1 ([7]). Let $E$ be a uniformly convex Banach space which satisfies the Opial's condition and let $C$ be a nonempty, closed and convex subset of $E$. Let $\alpha, \beta, \gamma, \delta \in \mathbb{R}$ and $\alpha^{\prime}, \beta^{\prime}, \gamma^{\prime}, \delta^{\prime} \in \mathbb{R}$. Let $S$ and $T$ be $(\alpha, \beta, \gamma, \delta)$ and $\left(\alpha^{\prime}, \beta^{\prime}, \gamma^{\prime} \delta^{\prime}\right)$-extended generalized hybrid mappings of $C$ into itself such that $\beta \leq 0$ and $\gamma \leq 0$ and $\beta^{\prime} \leq 0$ and $\gamma^{\prime} \leq 0$, respectively. Suppose that $F(S) \cap F(T) \neq \emptyset$. Let $\left\{x_{n}\right\}$ be a sequence in $C$ generated by $x_{1}=x \in C$ and

$$
x_{n+1}=\alpha_{n} x_{n}+\left(1-\alpha_{n}\right)\left(\gamma_{n} S x_{n}+\left(1-\gamma_{n}\right) T x_{n}\right), \quad \forall n \in \mathbb{N},
$$

where $a, b, c, d \in \mathbb{R},\left\{\gamma_{n}\right\}$ and $\left\{\alpha_{n}\right\}$ satisfy the following:

$$
0<a \leq \alpha_{n} \leq b<1 \text { and } 0<c \leq \gamma_{n} \leq d<1, \quad \forall n \in \mathbb{N} .
$$

Then, the sequence $\left\{x_{n}\right\}$ converges weakly to an element $z \in F(S) \cap F(T)$, where $F(S) \cap F(T)$ is the set of common fixed points of $S$ and $T$.

In this paper, we prove a weak convergence theorem of a Mann's type iteration for infinite families of extended generalized hybrid mappings in a Banach space satisfying Opial's condition. This theorem solves a problem posed by Hojo and Takahashi [7]. Using this result, we get new weak convergence theorems in a Banach space. In particular, we obtain a weak convergence theorem of the Mann's type iteration for finite families of extended generalized hybrid mappings in a Banach space.

\section{PRELIMINARIES}

Throughout this paper, we denote by $\mathbb{N}$ the set of positive integers and by $\mathbb{R}$ the set of real numbers. Let $E$ be a real Banach space with norm $\|\cdot\|$ and let $E^{*}$ be the topological dual space of $E$. We denote the value of $y^{*} \in E^{*}$ at $x \in E$ by $\left\langle x, y^{*}\right\rangle$. When $\left\{x_{n}\right\}$ is a sequence in $E$, we denote the strong convergence of $\left\{x_{n}\right\}$ to $x \in E$ by $x_{n} \rightarrow x$ and the weak convergence by $x_{n} \rightarrow x$. The modulus $\delta$ of convexity of $E$ is defined by

$$
\delta(\varepsilon)=\inf \left\{1-\frac{\|x+y\|}{2}:\|x\| \leq 1,\|y\| \leq 1,\|x-y\| \geq \varepsilon\right\}
$$

for all $\varepsilon$ with $0 \leq \varepsilon \leq 2$. A Banach space $E$ is said to be uniformly convex if $\delta(\varepsilon)>0$ for all $\varepsilon>0$. A uniformly convex Banach space is strictly convex and reflexive. Let $C$ be a nonempty subset of a Banach space $E$. A mapping $T: C \rightarrow E$ is nonexpansive if $\|T x-T y\| \leq\|x-y\|$ for all $x, y \in C$. A mapping $T: C \rightarrow E$ is quasi-nonexpansive if $F(T) \neq \emptyset$ and $\|T x-y\| \leq\|x-y\|$ for all $x \in C$ and $y \in F(T)$, where $F(T)$ is the set of fixed points of $T$. If $C$ is a nonempty, closed and convex subset of a strictly convex 
Banach space $E$ and $T: C \rightarrow E$ is quasi-nonexpansive, then $F(T)$ is closed and convex; see Itoh and Takahashi [10]. The duality mapping $J$ from $E$ into $2^{E^{*}}$ is defined by

$$
J x=\left\{x^{*} \in E^{*}:\left\langle x, x^{*}\right\rangle=\|x\|^{2}=\left\|x^{*}\right\|^{2}\right\}
$$

for all $x \in E$.

Lemma 2.1 ([17]). Let $E$ be a Banach space and let $J$ be the duality mapping on E. Then, for any $x, y \in E$,

$$
\|x\|^{2}-\|y\|^{2} \geq 2\langle x-y, j\rangle
$$

where $j \in J y$.

Let $E$ be a Banach space and let $A \subset E \times E$. Then, $A$ is accretive if for $\left(x_{1}, y_{1}\right),\left(x_{2}, y_{2}\right) \in A$, there exists $j \in J\left(x_{1}-x_{2}\right)$ such that $\left\langle y_{1}-y_{2}, j\right\rangle \geq 0$, where $J$ is the duality mapping of $E$. An accretive operator $A \subset E \times E$ is called $m$-accretive if $R(I+r A)=E$ for all $r>0$, where $I$ is the identity operator and $R(I+r A)$ is the range of $I+r A$. An accretive operator $A \subset E \times E$ is said to satisfy the range condition if $\overline{D(A)} \subset R(I+r A)$ for all $r>0$, where $\overline{D(A)}$ is the closure of the domain $D(A)$ of $A$. An $m$-accretive operator satisfies the range condition. If $C$ is a nonempty, closed and convex subset of a Banach space and $T$ is a nonexpansive mapping of $C$ into itself, then $A=I-T$ is an accretive operator and $C=D(A) \subset R(I+r A)$ for all $r>0$; see [17, Theorem 4.6.4].

Let $E$ be a Banach space and let $C$ be a nonempty subset of $E$. Then, a mapping $T: C \rightarrow E$ is said to be firmly nonexpansive [3] if

$$
\|T x-T y\|^{2} \leq\langle x-y, j\rangle
$$

for all $x, y \in C$, where $j \in J(T x-T y)$; see also [2,5]. It is known that the resolvent of an accretive operator satisfying the range condition in a Banach space is a firmly nonexpansive mapping of the closure of the domain into itself. In fact, let $C=\overline{D(A)}$ and $r>0$. Define the resolvent $J_{r}$ of $A$ as follows:

$$
J_{r} x=\{z \in D(A): x \in z+r A z\}
$$

for all $x \in \overline{D(A)}$. It is known that such $J_{r} x$ is a singleton; see [17]. We have that for $x_{1}, x_{2} \in \overline{D(A)}, x_{1}=$ $z_{1}+r y_{1}, y_{1} \in A z_{1}$ and $x_{2}=z_{2}+r y_{2}, y_{2} \in A z_{2}$. Since $A$ is accretive, we have that $\left\langle y_{1}-y_{2}, j\right\rangle \geq 0$, where $j \in J\left(z_{1}-z_{2}\right)$. So, we have

$$
\left\langle\frac{x_{1}-z_{1}}{r}-\frac{x_{2}-z_{2}}{r}, j\right\rangle \geq 0
$$

Furthermore,

$$
\begin{aligned}
\left\langle\frac{x_{1}-z_{1}}{r}\right. & \left.-\frac{x_{2}-z_{2}}{r}, j\right\rangle \geq 0 \\
& \Longleftrightarrow\left\langle x_{1}-z_{1}-\left(x_{2}-z_{2}\right), j\right\rangle \geq 0 \\
& \Longleftrightarrow\left\langle x_{1}-x_{2}, j\right\rangle \geq\left\|z_{1}-z_{2}\right\|^{2} .
\end{aligned}
$$

From $z_{1}=J_{r} x_{1}$ and $z_{2}=J_{r} x_{2}$, we have that $J_{r}$ is a firmly nonexpansive mapping of $C$ into itself; see also [3], [4] and [20]. Let $E$ be a Banach space and let $C$ be a nonempty subset of $E$. A mapping $T: C \rightarrow E$ is called extended generalized hybrid if it satisfies (1.3), that is, there are $\alpha, \beta, \gamma, \delta \in \mathbb{R}$ such that $\alpha+\beta+\gamma+\delta \geq 0, \alpha+\beta>0$ and

$$
\alpha\|T x-T y\|^{2}+\beta\|x-T y\|^{2}+\gamma\|T x-y\|^{2}+\delta\|x-y\|^{2} \leq 0
$$


for all $x, y \in C$. We call such a mapping $(\alpha, \beta, \gamma, \delta)$-extended generalized hybrid. We can also show that, in a Banach space, an $(\alpha, \beta, \gamma, \delta)$-extended generalized hybrid mapping is nonexpansive for $\alpha=1$, $\beta=\gamma=0$ and $\delta=-1$, nonspreading for $\alpha=2, \beta=\gamma=-1$ and $\delta=0$, and hybrid for $\alpha=3, \beta=\gamma=-1$ and $\delta=-1$. Nonexpansive mappings, nonspreading mappings and hybrid mappings in a Banach space are deduced from firmly nonexpansive mappings as follows: Let $T$ be a firmly nonexpansive mapping of $C$ into $E$. Then we have that for $x, y \in C$ and $j \in J(T x-T y)$,

$$
\|T x-T y\|^{2} \leq\langle x-y, j\rangle \text {. }
$$

From Theorem 2.1, we have that

$$
\begin{aligned}
\|T x-T y\|^{2} & \leq\langle x-y, j\rangle \\
& \Longleftrightarrow 0 \leq 2\langle x-T x-(y-T y), j\rangle \\
& \Longleftrightarrow 0 \leq\|x-y\|^{2}-\|T x-T y\|^{2} \\
& \Longleftrightarrow\|T x-T y\|^{2} \leq\|x-y\|^{2} .
\end{aligned}
$$

Futhermore, we have that, for $x, y \in C$ and $j \in J(T x-T y)$,

$$
\begin{aligned}
\|T x-T y\|^{2} & \leq\langle x-y, j\rangle \\
& \Longleftrightarrow 0 \leq 2\langle x-T x-(y-T y), j\rangle \\
& \Longleftrightarrow 0 \leq 2\langle x-T x, j\rangle+2\langle T y-y, j\rangle \\
& \Longrightarrow 0 \leq\|x-T y\|^{2}-\|T x-T y\|^{2}+\|T x-y\|^{2}-\|T x-T y\|^{2} \\
& \Longleftrightarrow 0 \leq\|x-T y\|^{2}+\|y-T x\|^{2}-2\|T x-T y\|^{2} \\
& \Longleftrightarrow 2\|T x-T y\|^{2} \leq\|x-T y\|^{2}+\|y-T x\|^{2} .
\end{aligned}
$$

Therefore, using (2.1) and (2.2), we have that

$$
\begin{aligned}
\|T x-T y\|^{2} & \leq\langle x-y, j\rangle \\
& \Longrightarrow 3\|T x-T y\|^{2} \leq\|x-T y\|^{2}+\|y-T x\|^{2}+\|x-y\|^{2} .
\end{aligned}
$$

Hojo and Takahashi [6] proved the following result.

Lemma 2.2 ([6]). Let $E$ be a Banach space, let $C$ be a nonempty, closed and convex subset of $E$. Then an extended generalized hybrid mapping which has a fixed point is quasi-nonexpansive.

The following result was proved by $\mathrm{Xu}$ [21].

Lemma 2.3 ([21]). Let $E$ be a uniformly convex Banach space and let $r>0$. Then there exists a strictly increasing, continuous and convex function $g:[0, \infty) \rightarrow[0, \infty)$ such that $g(0)=0$ and

$$
\|\mu x+(1-\mu) y\|^{2} \leq \mu\|x\|^{2}+(1-\mu)\|y\|^{2}-\mu(1-\mu) g(\|x-y\|)
$$

for all $x, y \in B_{r}$ and $\mu$ with $0 \leq \mu \leq 1$, where $B_{r}=\{z \in E:\|z\| \leq r\}$.

Let $E$ be a Banach space. Then, $E$ satisfies the Opial's condition [15] if, for any $\left\{x_{n}\right\}$ of $E$ such that $x_{n} \rightarrow x$ and $x \neq y$,

$$
\liminf _{n \rightarrow \infty}\left\|x_{n}-x\right\|<\liminf _{n \rightarrow \infty}\left\|x_{n}-y\right\| .
$$


Let $E$ be a Banach space. Let $C$ be a nonempty, closed and convex subset of $E$. Let $T: C \rightarrow E$ be a mapping. Then, $p \in C$ is called an asymptotic fixed point of $T$ [16] if there exists $\left\{x_{n}\right\} \subset C$ such that $x_{n} \rightarrow p$ and $\lim _{n \rightarrow \infty}\left\|x_{n}-T x_{n}\right\|=0$. We denote by $\hat{F}(T)$ the set of asymptotic fixed points of $T$. A mapping $T: C \rightarrow E$ is said to be demiclosed if $\hat{F}(T)=F(T)$. We know the following result from Hojo and Takahashi [6].

Lemma 2.4 ([6]). Let $E$ be a Banach space satisfying Opial's condition and let $C$ be a nonempty, closed and convex subset of $E$. Let $\alpha, \beta, \gamma, \delta \in \mathbb{R}$ and let $T$ be an $(\alpha, \beta, \gamma, \delta)$-extended generalized hybrid mapping of $C$ into $E$ which satisfies $\beta \leq 0$ and $\gamma \leq 0$. Then $\hat{F}(T)=F(T)$, i.e., $T$ is demiclosed.

If $E$ is a Banach space satisfying the Opial's condition, then nonexpansive mappings, nonspreading mappngs and hybrid mappings are demiclosed; see [6].

\section{WeAK Convergence TheOrems}

In this section, we first prove a weak convergence theorem of Mann's type iteration [14] for an infinite family of extended generalized hybrid mappings in a Banach space satisfying the Opial's condition.

Theorem 3.1. Let $E$ be a uniformly convex Banach space which satisfies the Opial's condition and let $C$ be a nonempty, closed and convex subset of $E$. Let $\alpha_{j}, \beta_{j}, \gamma_{j}, \delta_{j} \in \mathbb{R}$ for all $j \in \mathbb{N}$ and let $\left\{T_{j}\right\}$ be a sequence of $\left(\alpha_{j}, \beta_{j}, \gamma_{j}, \delta_{j}\right)$-extended generalized hybrid mappings of $C$ into itself such that $\beta_{j} \leq 0$ and $\gamma_{j} \leq 0$ for all $j \in \mathbb{N}$. Suppose that $\cap_{j=1}^{\infty} F\left(T_{j}\right) \neq \emptyset$. Let $\left\{x_{n}\right\}$ be a sequence in $C$ generated by $x_{1}=x \in C$ and

$$
x_{n+1}=\alpha_{n} x_{n}+\left(1-\alpha_{n}\right) \sum_{j=1}^{\infty} \xi_{j} T_{j} x_{n}, \quad \forall n \in \mathbb{N},
$$

where $a, b \in \mathbb{R}$ and $\left\{\xi_{j}\right\},\left\{\alpha_{n}\right\} \subset(0,1)$ satisfy the following:

(1) $\sum_{j=1}^{\infty} \xi_{j}=1$;

(2) $0<a \leq \alpha_{n} \leq b<1, \quad \forall n \in \mathbb{N}$.

Then, the sequence $\left\{x_{n}\right\}$ converges weakly to an element $z \in \cap_{j=1}^{\infty} F\left(T_{j}\right)$.

Proof. Since $T_{j}$ is quasi-nonexpansive from Lemma 2.2, we have that $F\left(T_{j}\right)$ is closed and convex. Therefore, $\cap_{j=1}^{\infty} F\left(T_{j}\right)$ is closed and convex. Put

$$
T x=\sum_{j=1}^{\infty} \xi_{j} T_{j} x
$$

for all $x \in C$; see Bruck [3]. Then we can prove that $T$ is well defined. In fact, since $T_{j}$ is quasinonexpansive, we have that, for any $y_{0} \in \cap_{j=1}^{\infty} F\left(T_{j}\right)$,

$$
\left\|T_{j} x\right\| \leq\left\|T_{j} x-y_{0}\right\|+\left\|y_{0}\right\| \leq\left\|x-y_{0}\right\|+\left\|y_{0}\right\| .
$$

Thus $T x=\sum_{j=1}^{\infty} \xi_{j} T_{j} x$ converges absolutely for all $x$ in $C$. Furthermore, we have

$$
F(T)=\cap_{j=1}^{\infty} F\left(T_{j}\right) .
$$

In fact, it is obvious that $\cap_{j=1}^{\infty} F\left(T_{j}\right) \subset F(T)$. We show that $F(T) \subset \cap_{j=1}^{\infty} F\left(T_{j}\right)$. For $y_{0} \in \cap_{j=1}^{\infty} F\left(T_{j}\right)$ and $w \in F(T)$, we have that

$$
w-y_{0}=T w-y_{0}=\sum_{j=1}^{\infty} \xi_{j} T_{j} w-y_{0}=\sum_{j=1}^{\infty} \xi_{j}\left(T_{j} w-y_{0}\right)
$$


and hence

$$
\left\|w-y_{0}\right\| \leq \sum_{j=1}^{\infty} \xi_{j}\left\|T_{j} w-y_{0}\right\| \leq \sum_{j=1}^{\infty} \xi_{j}\left\|w-y_{0}\right\|=\left\|w-y_{0}\right\| .
$$

Then, $\left\|T_{j} w-y_{0}\right\|=\left\|w-y_{0}\right\|$ for all $j \in \mathbb{N}$. Assume that $T_{1} w-y_{0} \neq T_{i} w-y_{0}$ for some $i \in \mathbb{N}$. Since $E$ is strictly convex, there exists $\delta>0$ such that

$$
\left\|\tau\left(T_{1} w-y_{0}\right)+(1-\tau)\left(T_{i} w-y_{0}\right)\right\|=\left\|w-y_{0}\right\|-\delta,
$$

where $\tau=\frac{\xi_{1}}{\xi_{1}+\xi_{i}}$. Then, we have that, for any $m \in \mathbb{N}$ with $i \leq m$,

$$
\begin{aligned}
& \left\|\sum_{j=1}^{m} \xi_{j}\left(T_{j} w-y_{0}\right)\right\| \\
& \quad=\left\|\left(\xi_{1}+\xi_{i}\right)\left(\tau\left(T_{1} w-y_{0}\right)+(1-\tau)\left(T_{i} w-y_{0}\right)\right)+\sum_{j \neq 1, i}^{m} \xi_{j}\left(T_{j} w-y_{0}\right)\right\| \\
& \quad \leq\left(\xi_{1}+\xi_{i}\right)\left\|\tau\left(T_{1} w-y_{0}\right)+(1-\tau)\left(T_{i} w-y_{0}\right)\right\|+\sum_{j \neq 1, i}^{m} \xi_{j}\left\|T_{j} w-y_{0}\right\| \\
& \quad \leq\left(\xi_{1}+\xi_{i}\right)\left(\left\|w-y_{0}\right\|-\delta\right)+\sum_{j \neq 1, i}^{m} \xi_{j}\left\|w-y_{0}\right\| \\
& \quad=\sum_{j=1}^{m} \xi_{j}\left\|w-y_{0}\right\|-\left(\xi_{1}+\xi_{i}\right) \delta \\
& =\left\|w-y_{0}\right\|-\left(\xi_{1}+\xi_{i}\right) \delta .
\end{aligned}
$$

Since $w-y_{0}=\sum_{j=1}^{\infty} \xi_{j}\left(T_{j} w-y_{0}\right)$, we have that

$$
\left\|w-y_{0}\right\| \leq\left\|w-y_{0}\right\|-\left(\xi_{1}+\xi_{i}\right) \delta .
$$

This is a contradiction. Therefore, we have that $T_{1} w-y_{0}=T_{i} w-y_{0}$ for all $i \in \mathbb{N}$. From $w-y_{0}=$ $\sum_{j=1}^{\infty} \xi_{j}\left(T_{j} w-y_{0}\right)$, we have that $w-y_{0}=T_{i} w-y_{0}$ for all $i \in \mathbb{N}$ and hence $w=T_{i} w$ for all $i \in \mathbb{N}$. This implies that $F(T) \subset \cap_{j=1}^{\infty} F\left(T_{j}\right)$.

Let $w \in \cap_{j=1}^{\infty} F\left(T_{j}\right)$. We have that

$$
\begin{aligned}
\left\|T x_{n}-w\right\| & =\left\|\sum_{j=1}^{\infty} \xi_{j} T_{j} x_{n}-w\right\| \leq \sum_{j=1}^{\infty} \xi_{j}\left\|T_{j} x_{n}-w\right\| \\
& \leq \sum_{j=1}^{\infty} \xi_{j}\left\|x_{n}-w\right\|=\left\|x_{n}-w\right\| .
\end{aligned}
$$

Using (3.1), we have that

$$
\begin{aligned}
\left\|x_{n+1}-w\right\| & =\left\|\alpha_{n} x_{n}+\left(1-\alpha_{n}\right) T x_{n}-w\right\| \\
& \leq \alpha_{n}\left\|x_{n}-w\right\|+\left(1-\alpha_{n}\right)\left\|T x_{n}-w\right\| \\
& \leq \alpha_{n}\left\|x_{n}-w\right\|+\left(1-\alpha_{n}\right)\left\|x_{n}-w\right\| \\
& =\left\|x_{n}-w\right\| .
\end{aligned}
$$

Then, $\lim _{n \rightarrow \infty}\left\|x_{n}-w\right\|$ exists. Thus we have that the sequence $\left\{x_{n}\right\}$ is bounded. This implies that $\left\{T x_{n}\right\}$ is bounded. Let

$$
r=\max \left\{\sup _{n \in \mathbb{N}}\left\|x_{n}-w\right\|, \sup _{n \in \mathbb{N}}\left\|T x_{n}-w\right\|\right\}
$$


From Lemma 2.3, there exists a strictly increasing, continuous and convex function $g:[0, \infty) \rightarrow[0, \infty)$ such that $g(0)=0$ and

$$
\|\mu x+(1-\mu) y\|^{2} \leq \mu\|x\|^{2}+(1-\mu)\|y\|^{2}-\mu(1-\mu) g(\|x-y\|)
$$

for all $x, y \in B_{r}$ and $\mu$ with $0 \leq \mu \leq 1$, where $B_{r}=\{z \in E:\|z\| \leq r\}$. Then we have that for $w \in \cap_{j=1}^{\infty} F\left(T_{j}\right)$ and $n \in \mathbb{N}$,

$$
\begin{aligned}
\left\|x_{n+1}-w\right\|^{2} & =\left\|\alpha_{n} x_{n}+\left(1-\alpha_{n}\right) T x_{n}-w\right\|^{2} \\
& =\left\|\alpha_{n}\left(x_{n}-w\right)+\left(1-\alpha_{n}\right)\left(T x_{n}-w\right)\right\|^{2} \\
& \leq \alpha_{n}\left\|x_{n}-w\right\|^{2}+\left(1-\alpha_{n}\right)\left\|T x_{n}-w\right\|^{2}-\alpha_{n}\left(1-\alpha_{n}\right) g\left(\left\|x_{n}-T x_{n}\right\|\right) \\
& \leq \alpha_{n}\left\|x_{n}-w\right\|^{2}+\left(1-\alpha_{n}\right)\left\|x_{n}-w\right\|^{2}-\alpha_{n}\left(1-\alpha_{n}\right) g\left(\left\|x_{n}-T x_{n}\right\|\right) \\
& =\left\|x_{n}-w\right\|^{2}-\alpha_{n}\left(1-\alpha_{n}\right) g\left(\left\|x_{n}-T x_{n}\right\|\right)
\end{aligned}
$$

and hence

$$
\alpha_{n}\left(1-\alpha_{n}\right) g\left(\left\|x_{n}-T x_{n}\right\|\right) \leq\left\|x_{n}-w\right\|^{2}-\left\|x_{n+1}-w\right\|^{2}
$$

Since $\lim _{n \rightarrow \infty}\left\|x_{n}-w\right\|^{2}$ exists, we have from $0<a \leq \alpha_{n} \leq b<1$ that

$$
\lim _{n \rightarrow \infty} g\left(\left\|x_{n}-T x_{n}\right\|\right)=0
$$

From the properties of $g$, we have

$$
\lim _{n \rightarrow \infty}\left\|x_{n}-T x_{n}\right\|=0
$$

For any $\varepsilon>0$ and $i, k \in \mathbb{N}$ with $i \neq k$, we take $m \in \mathbb{N}$ such that $i, k \leq m$ and $\left\|\sum_{j=m+1}^{\infty} \xi_{j}\left(T_{j} x_{n}-w\right)\right\| \leq \varepsilon$, where $w \in \cap_{i=1}^{\infty} F\left(T_{j}\right)$. Using this, we have from Lemma 2.1 that, for $j\left(x_{n}-w\right) \in J\left(x_{n}-w\right)$,

$$
\begin{aligned}
\left\|x_{n}-w\right\|^{2} & =\left\|x_{n}-T x_{n}+T x_{n}-w\right\|^{2} \\
& \leq\left\|T x_{n}-w\right\|^{2}+2\left\langle x_{n}-T x_{n}, j\left(x_{n}-w\right)\right\rangle \\
& =\left\|\sum_{j=1}^{\infty} \xi_{j} T_{j} x_{n}-w\right\|^{2}+2\left\langle x_{n}-T x_{n}, j\left(x_{n}-w\right)\right\rangle \\
& =\left\|\sum_{j=1}^{\infty} \xi_{j}\left(T_{j} x_{n}-w\right)\right\|^{2}+2\left\langle x_{n}-T x_{n}, j\left(x_{n}-w\right)\right\rangle \\
& \leq\left(\left\|\sum_{j=1}^{m} \xi_{j}\left(T_{j} x_{n}-w\right)\right\|+\varepsilon\right)^{2}+2\left\langle x_{n}-T x_{n}, j\left(x_{n}-w\right)\right\rangle \\
& \leq\left\|\sum_{j=1}^{m} \xi_{j}\left(T_{j} x_{n}-w\right)\right\|^{2}+2 r \varepsilon+\varepsilon^{2}+2\left\langle x_{n}-T x_{n}, j\left(x_{n}-w\right)\right\rangle .
\end{aligned}
$$


Set $\sigma=\frac{\xi_{i}}{\xi_{i}+\xi_{k}}$. Then $1-\sigma=\frac{\xi_{k}}{\xi_{i}+\xi_{k}}$. We have that, for $w \in \cap_{j=1}^{\infty} F\left(T_{j}\right)$,

$$
\begin{aligned}
&\left\|\sum_{j=1}^{m} \xi_{j}\left(T_{j} x_{n}-w\right)\right\|^{2} \\
&=\left\|\left(\xi_{i}+\xi_{k}\right)\left(\sigma\left(T_{i} x_{n}-w\right)+(1-\sigma)\left(T_{k} x_{n}-w\right)\right)+\sum_{j \neq i, k}^{m} \xi_{j}\left(T_{j} x_{n}-w\right)\right\|^{2} \\
& \leq\left(\xi_{i}+\xi_{k}\right)\left\|\sigma\left(T_{i} x_{n}-w\right)+(1-\sigma)\left(T_{k} x_{n}-w\right)\right\|^{2}+\sum_{j \neq i, k}^{m} \xi_{j}\left\|T_{j} x_{n}-w\right\|^{2} \\
& \leq\left(\xi_{i}+\xi_{k}\right)\left(\sigma\left\|T_{i} x_{n}-w\right\|^{2}+(1-\sigma)\left\|T_{k} x_{n}-w\right\|^{2}\right) \\
&-\left(\xi_{i}+\xi_{k}\right) \sigma(1-\sigma) g\left(\left\|T_{i} x_{n}-T_{k} x_{n}\right\|\right)+\sum_{j \neq i, k}^{m} \xi_{j}\left\|T_{j} x_{n}-w\right\|^{2} \\
& \leq\left(\xi_{i}+\xi_{k}\right)\left(\sigma\left\|x_{n}-w\right\|^{2}+(1-\sigma)\left\|x_{n}-w\right\|^{2}\right) \\
&-\left(\xi_{i}+\xi_{k}\right) \sigma(1-\sigma) g\left(\left\|T_{i} x_{n}-T_{k} x_{n}\right\|\right)+\sum_{j \neq i, k}^{m} \xi_{j}\left\|x_{n}-w\right\|^{2} \\
& \leq \sum_{j=1}^{m} \xi_{j}\left\|x_{n}-w\right\|^{2}-\left(\xi_{i}+\xi_{k}\right) \sigma(1-\sigma) g\left(\left\|T_{i} x_{n}-T_{k} x_{n}\right\|\right) \\
& \leq\left\|x_{n}-w\right\|^{2}-\left(\xi_{i}+\xi_{k}\right) \sigma(1-\sigma) g\left(\left\|T_{i} x_{n}-T_{k} x_{n}\right\|\right) .
\end{aligned}
$$

Then, we have

$$
\begin{aligned}
\left\|x_{n}-w\right\|^{2}= & \left\|x_{n}-T x_{n}+T x_{n}-w\right\|^{2} \\
\leq & \left\|x_{n}-w\right\|^{2}-\left(\xi_{i}+\xi_{k}\right) \sigma(1-\sigma) g\left(\left\|T_{i} x_{n}-T_{k} x_{n}\right\|\right) \\
& +2\left\langle x_{n}-T x_{n}, j\left(x_{n}-w\right)\right\rangle+2 r \varepsilon+\varepsilon^{2}
\end{aligned}
$$

and hence

$$
\left(\xi_{i}+\xi_{k}\right) \sigma(1-\sigma) g\left(\left\|T_{i} x_{n}-T_{k} x_{n}\right\|\right) \leq 2\left\langle x_{n}-T x_{n}, j\left(x_{n}-w\right)\right\rangle+2 r \varepsilon+\varepsilon^{2} .
$$

Since $\lim _{n \rightarrow \infty}\left\|x_{n}-T x_{n}\right\|=0$, we have that

$$
\limsup _{n \rightarrow \infty} g\left(\left\|T_{i} x_{n}-T_{k} x_{n}\right\|\right) \leq 2 \varepsilon+\varepsilon^{2} .
$$

Since $\varepsilon>0$ is arbitrary, we have that $g\left(\left\|T_{i} x_{n}-T_{k} x_{n}\right\|\right) \rightarrow 0$. It follows that

$$
\left\|T_{i} x_{n}-T_{k} x_{n}\right\| \rightarrow 0
$$

For any $\varepsilon>0$ and $i \in \mathbb{N}$, we take $m \in \mathbb{N}$ such that $\left\|\sum_{j=m+1}^{\infty} \xi_{j}\left(T_{j} x_{n}-T_{i} x_{n}\right)\right\| \leq \varepsilon$. Then we have that

$$
\begin{aligned}
\left\|x_{n}-T_{i} x_{n}\right\| & =\left\|x_{n}-T x_{n}+T x_{n}-T_{i} x_{n}\right\| \\
& \leq\left\|x_{n}-T x_{n}\right\|+\left\|T x_{n}-T_{i} x_{n}\right\| \\
& =\left\|x_{n}-T x_{n}\right\|+\left\|\sum_{j=1}^{\infty} \xi_{j}\left(T_{j} x_{n}-T_{i} x_{n}\right)\right\| \\
& \leq\left\|x_{n}-T x_{n}\right\|+\left\|\sum_{j=1}^{m} \xi_{j}\left(T_{j} x_{n}-T_{i} x_{n}\right)\right\|+\varepsilon .
\end{aligned}
$$


From $\left\|x_{n}-T x_{n}\right\| \rightarrow 0$ and $T_{j} x_{n}-T_{i} x_{n} \rightarrow 0$ for all $j \in \mathbb{N}$, we have that

$$
\limsup _{n \rightarrow \infty}\left\|x_{n}-T_{i} x_{n}\right\| \leq \varepsilon
$$

Since $\varepsilon>0$ is arbitrary, we have that

$$
\left\|x_{n}-T_{i} x_{n}\right\| \rightarrow 0, \quad \forall i \in \mathbb{N} .
$$

Since $\left\{x_{n}\right\}$ is bounded, there exists a subsequence $\left\{x_{n_{i}}\right\}$ of $\left\{x_{n}\right\}$ such that $x_{n_{i}} \rightarrow v$ for some $v \in C$. From (3.4) and Lemma 2.4, we have that $v$ is a point of $\cap_{j=1}^{\infty} F\left(T_{j}\right)$. Let $\left\{x_{n_{i}}\right\}$ and $\left\{x_{n_{j}}\right\}$ be two subsequences of $\left\{x_{n}\right\}$ such that $x_{n_{i}} \rightarrow u$ and $x_{n_{j}} \rightarrow v$. We have that $u, v \in \cap_{j=1}^{\infty} F\left(T_{j}\right)$. Suppose $u \neq v$. From $u, v \in$ $\cap_{j=1}^{\infty} F\left(T_{j}\right)$, we know that $\lim _{n \rightarrow \infty}\left\|x_{n}-u\right\|$ and $\lim _{n \rightarrow \infty}\left\|x_{n}-v\right\|$ exist. Since $E$ satisfies the Opial's condition, we have that

$$
\begin{gathered}
\lim _{n \rightarrow \infty}\left\|x_{n}-u\right\|=\lim _{i \rightarrow \infty}\left\|x_{n_{i}}-u\right\|<\lim _{i \rightarrow \infty}\left\|x_{n_{i}}-v\right\| \\
=\lim _{n \rightarrow \infty}\left\|x_{n}-v\right\|=\lim _{j \rightarrow \infty}\left\|x_{n_{j}}-v\right\| \\
<\lim _{j \rightarrow \infty}\left\|x_{n_{j}}-u\right\|=\lim _{n \rightarrow \infty}\left\|x_{n}-u\right\| .
\end{gathered}
$$

This is a contradiction. Thus we must have $u=v$. This implies that $\left\{x_{n}\right\}$ converges weakly to a point of $\cap_{j=1}^{\infty} F\left(T_{j}\right)$. This completes the proof.

Using Theorem 3.1, we obtain the following weak convergence theorem for a finite family of extended generalized hybrid mappings in a Banach space satisfying the Opial's condition; see Hojo and Takahashi [6] for two extended generalized hybrid mappings.

Theorem 3.2 ([6]). Let E be a uniformly convex Banach space which satisfies the Opial's condition and let $C$ be a nonempty, closed and convex subset of $E$. Let $\alpha_{j}, \beta_{j}, \gamma_{j}, \delta_{j} \in \mathbb{R}$ for all $j \in\{1,2, \ldots, M\}$ and let $\left\{T_{j}\right\}_{j=1}^{M}$ be a finite family of $\left(\alpha_{j}, \beta_{j}, \gamma_{j}, \delta_{j}\right)$-extended generalized hybrid mappings of $C$ into itself such that $\beta_{j} \leq 0$ and $\gamma_{j} \leq 0$ for all $j \in\{1,2, \ldots, M\}$. Suppose that $\cap_{j=1}^{M} F\left(T_{j}\right) \neq \emptyset$. Let $\left\{x_{n}\right\}$ be a sequence generated by $x_{1}=x \in C$ and

$$
x_{n+1}=\alpha_{n} x_{n}+\left(1-\alpha_{n}\right) \sum_{j=1}^{M} \xi_{j} T_{j} x_{n}, \quad \forall n \in \mathbb{N},
$$

where $a, b \in \mathbb{R}$ and $\left\{\xi_{j}\right\},\left\{\alpha_{n}\right\} \subset(0,1)$ satisfy the following:

(1) $\sum_{j=1}^{M} \xi_{j}=1$;

(2) $0<a \leq \alpha_{n} \leq b<1, \quad \forall n \in \mathbb{N}$.

Then, the sequence $\left\{x_{n}\right\}$ converges weakly to an element $z \in \cap_{j=1}^{M} F\left(T_{j}\right)$.

Proof. From $\xi_{M} T_{M}=\frac{\xi_{M}}{2} T_{M}+\frac{\xi_{M}}{2^{2}} T_{M}+\cdots$, we have that

$$
\sum_{j=1}^{M} \xi_{j} T_{j}=\sum_{j=1}^{M-1} \xi_{j} T_{j}+\frac{\xi_{M}}{2} T_{M}+\frac{\xi_{M}}{2^{2}} T_{M}+\cdots
$$

Thus, we have the desired result from Theorem 3.1.

Using Theorem 3.2, we obtain the following result. 
Theorem 3.3. Let $E$ be a uniformly convex Banach space which satisfies the Opial's condition and let $C$ be a nonempty, closed and convex subset of $E$. Let $\alpha_{j}, \beta_{j}, \gamma_{j}, \delta_{j} \in \mathbb{R}$ for all $j \in\{1,2, \ldots, M\}$ and let $\left\{T_{j}\right\}_{j=1}^{M}$ be a finite family of $\left(\alpha_{j}, \beta_{j}, \gamma_{j}, \delta_{j}\right)$-extended generalized hybrid mappings of $C$ into itself such that $\beta_{j} \leq 0$ and $\gamma_{j} \leq 0$ for all $j \in\{1,2, \ldots, M\}$. Suppose that $\cap_{j=1}^{M} F\left(T_{j}\right) \neq \emptyset$. Let $\lambda$ be a real number with $0<\lambda<1$. Define a mapping $U: C \rightarrow C$ by

$$
U=\lambda I+(1-\lambda) \sum_{j=1}^{M} \xi_{j} T_{j},
$$

where $\left\{\xi_{j}\right\} \subset(0,1)$ satisfies $\sum_{j=1}^{M} \xi_{j}=1$. Then for any $x \in C, U^{n} x$ converges weakly to an element $z \in \cap_{j=1}^{M} F\left(T_{j}\right)$.

Proof. Putting $\alpha_{n}=\lambda$ for all $n \in \mathbb{N}$ in Theorem 3.2, we have that, for any $x_{1}=x \in C$,

$$
x_{2}=U x_{1}=U x, x_{3}=U^{2} x_{1}=U^{2} x \ldots
$$

Thus we have from Theorem 3.2 that $U^{n} x$ converges weakly to an element $z \in \cap_{j=1}^{M} F\left(T_{j}\right)$. This completes the proof.

Using Theorem 3.2, we also obtain the following result [6].

Theorem 3.4 ([6]). Let $E$ be a uniformly convex Banach space which satisfies the Opial's condition and let $C$ be a nonempty, closed and convex subset of $E$. Let $\alpha, \beta, \gamma, \delta \in \mathbb{R}$ and let $T$ be an $(\alpha, \beta, \gamma, \delta)$ extended generalized hybrid mapping of $C$ into itself such that $\beta \leq 0$ and $\gamma \leq 0$. Let $\left\{\alpha_{n}\right\}$ be a sequence of real numbers such that $0<a \leq \alpha_{n} \leq b<1$ for some $a, b \in \mathbb{R}$ and define a sequence $\left\{x_{n}\right\}$ of $C$ as follows: $x_{1}=x \in C$ and

$$
x_{n+1}=\alpha_{n} x_{n}+\left(1-\alpha_{n}\right) T x_{n}, \quad \forall n \in \mathbb{N} .
$$

If $F(T) \neq \emptyset$, then $\left\{x_{n}\right\}$ converges weakly to some element $z \in F(T)$.

Proof. Putting $T=T_{1}=T_{2}=\cdots=T_{M}$ and $\xi_{j}=\frac{1}{M}$ in Theorem 3.2, we obtain the desired result from Theorem 3.2.

Using Theorems 3.1 and 3.2, we can also prove the following weak convergence theorems for families of nonexpansive mappings and nonspreading mappings in a Banach space.

Theorem 3.5. Let $E$ be a uniformly convex Banach space which satisfies the Opial's condition and let $C$ be a nonempty, closed and convex subset of $E$. Let $\left\{T_{j}\right\}$ be a sequence of nonexpansive mappings of $C$ into itself. Let $\left\{\xi_{j}\right\}$ be a family of real numbers in $(0,1)$ such that $\sum_{j=1}^{\infty} \xi_{j}=1$. Suppose that

$$
\Omega:=\cap_{j=1}^{\infty} F\left(T_{j}\right) \neq \emptyset .
$$

Let $\left\{x_{n}\right\}$ be a sequence in $C$ generated by $x_{1}=x \in C$ and

$$
x_{n+1}=\lambda_{n} x_{n}+\left(1-\lambda_{n}\right) \sum_{j=1}^{\infty} \xi_{j} T_{j} x_{n}, \quad \forall n \in \mathbb{N},
$$

where $a, b \in \mathbb{R}$ and $\left\{\lambda_{n}\right\} \subset(0,1)$ satisfy the following:

$$
0<a \leq \lambda_{n} \leq b<1, \quad \forall n \in \mathbb{N} .
$$

Then, $\left\{x_{n}\right\}$ converges weakly to an element $z \in \Omega$. 
Proof. Nonexpansive mappings are contained in the class of extended generalized hybrid mappings satisfying the conditions of Theorem 3.1. In particular, nonexpansive mappings in a Banach space satisfying Opial's condition are quasi-nonexpansive and demiclosed. Then, we obtain the desired result from Theorem 3.1.

Theorem 3.6. Let $E$ be a uniformly convex Banach space which satisfies the Opial's condition and let $C$ be a nonempty, closed and convex subset of E. Let $\left\{T_{j}\right\}_{j=1}^{M}$ be a sequence of nonspreading mappings of $C$ into itself. Let $\left\{\xi_{j}\right\}$ be a family of real numbers in $(0,1)$ such that $\sum_{j=1}^{M} \xi_{j}=1$. Suppose that

$$
\Omega:=\cap_{j=1}^{M} F\left(T_{j}\right) \neq \emptyset .
$$

Let $\left\{x_{n}\right\}$ be a sequence generated by $x_{1}=x \in C$ and

$$
x_{n+1}=\lambda_{n} x_{n}+\left(1-\lambda_{n}\right) \sum_{j=1}^{M} \xi_{j} T_{j} x_{n}, \quad \forall n \in \mathbb{N},
$$

where $a, b \in \mathbb{R}$ and $\left\{\lambda_{n}\right\} \subset(0,1)$ satisfy the following:

$$
0<a \leq \lambda_{n} \leq b<1, \quad \forall n \in \mathbb{N} .
$$

Then, $\left\{x_{n}\right\}$ converges weakly to an element $z \in \Omega$.

Proof. Nonspreading mappings are contained in the class of extended generalized hybrid mappings satisfying the conditions of Theorem 3.2. In particular, nonspreading mappings in a Banach space satisfying Opial's condition are quasi-nonexpansive and demiclosed. Then, we obtain from Theorem 3.2 the desired result.

\section{Acknowledgment}

The author is grateful to the reviewers for useful suggestions which improve the contents of this paper.

\section{REFERENCES}

[1] K. Aoyama, S. Iemoto, F. Kohsaka, W. Takahashi, Fixed point and ergodic theorems for $\lambda$-hybrid mappings in Hilbert spaces, J. Nonlinear Convex Anal. 11 (2010), 335-343.

[2] F.E. Browder, Convergence theorems for sequences of nonlinear operators in Banach spaces, Math. Z. 100 (1967), 201225.

[3] R.E. Bruck, Nonexpansive projections on subsets of Banach spaces, Pacific J. Math. 47 (1973), 341-355.

[4] R.E. Bruck, S. Reich, Nonexpansive projections and resolvents of accretive operators in Banach spaces, Houston J. Math. 3 (1977), 459-470.

[5] K. Goebel, W. A. Kirk, Topics in Metric Fixed Point Theory, Cambridge University Press, Cambridge, 1990.

[6] M. Hojo, W. Takahashi, Fixed point and weak convergence theorems for nonlinear hybrid mappings in Banach spaces, Linear Nonlinear Anal. 3 (2017), 61-72.

[7] M. Hojo, W. Takahashi, Fixed point and weak convergence theorems for noncommutative two extended generalized hybrid mappings in Banach spaces, to appear.

[8] M. Hojo, W. Takahashi, J.-C. Yao, Weak and strong mean convergence theorems for super hybrid mappings in Hilbert spaces, Fixed Point Theory 12 (2011), 113-126.

[9] T. Igarashi, W. Takahashi, K. Tanaka, Weak convergence theorems for nonspreading mappings and equilibrium problems, in Nonlinear Analysis and Optimization (S. Akashi, W. Takahashi and T. Tanaka Eds.), Yokohama Publishers, Yokohama, 2008, pp. 75-85. 
[10] S. Itoh, W. Takahashi, The common fixed point theory of single-valued mappings and multi-valued mappings, Pacific J. Math. 79 (1978), 493-508.

[11] P. Kocourek, W. Takahashi, J. -C. Yao, Fixed point theorems and weak convergence theorems for generalized hybrid mappings in Hilbert spaces, Taiwanese J. Math. 14 (2010), 2497-2511.

[12] F. Kohsaka, W. Takahashi, Existence and approximation of fixed points of firmly nonexpansive-type mappings in Banach spaces, SIAM J. Optim. 19 (2008), 824-835.

[13] F. Kohsaka, W. Takahashi, Fixed point theorems for a class of nonlinear mappings related to maximal monotone operators in Banach spaces, Arch. Math. 91 (2008), 166-177.

[14] W.R. Mann, Mean value methods in iteration, Proc. Amer. Math. Soc. 4 (1953), 506-510.

[15] Z. Opial, Weak convergence of the sequence of successive approximations for nonexpansive mappings, Bull. Amer. Math. Soc. 73 (1967), 591-597.

[16] S. Reich, A weak convergence theorem for the alternating method with Bregman distances, in Theory and Applications of Nonlinear Operators of Accretive and Monotone Type (A. G. Kartsatos Ed.), Marcel Dekker, New York, 1996, pp. 313-318.

[17] W. Takahashi, Nonlinear Functional Analysis, Yokohoma Publishers, Yokohoma, 2000.

[18] W. Takahashi, Fixed point theorems for new nonlinear mappings in a Hilbert space, J. Nonlinear Convex Anal. 11 (2010), 79-88.

[19] W. Takahashi, Weak and strong convergence theorems for noncommutative two generalized hybrid mappings in Hilbert spaces, J. Nonlinear Convex Anal. 19 (2018), 867-880.

[20] W. Takahashi, J.-C. Yao, Nonlinear operators of monotone type and convergence theorems with equilibrium problems in Banach spaces, Taiwanese J. Math. 15 (2011), 787-818.

[21] H.K. Xu, Inequalities in Banach spaces with applications, Nonlinear Anal. 16 (1981), 1127-1138. 